\title{
Improved delivery of PLGA microparticles and microparticle-cell scaffolds in clinical needle gauges using modified viscosity formulations
}

\author{
Omar Qutachi ${ }^{\mathrm{a}}$, Emma J. Wright ${ }^{\mathrm{a}, \mathrm{b}}$, Gemma Bray ${ }^{\mathrm{a}}$, Omar A. Hamid ${ }^{\mathrm{a}}$, Felicity R.A.J. Rose ${ }^{\mathrm{a}}$, \\ Kevin M. Shakesheff ${ }^{a}$, Derfogail Delcassian ${ }^{\mathrm{a}, \mathrm{c}, *}$

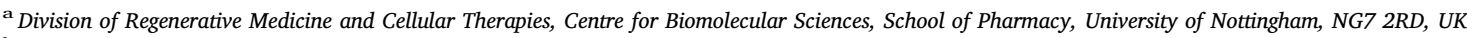 \\ ${ }^{\mathrm{b}}$ School of Pharmacy, University of Lincoln, LN6 7DL, UK \\ ${ }^{\mathrm{c}}$ David H. Koch Institute for Integrative Cancer Research, MIT, 02140 MA, USA
}

\section{A R T I C L E I N F O}

\section{Keywords:}

High viscosity formulation

Microparticle delivery

Cell particle scaffolds

Needle gauge

\begin{abstract}
A B S T R A C T
Polymer microparticles are widely used as acellular drug delivery platforms in regenerative medicine, and have emerging potential as cellular scaffolds for therapeutic cell delivery. In the clinic, PLGA microparticles are typically administered intramuscularly or subcutaneously, with the clinician and clinical application site determining the precise needle gauge used for delivery. Here, we explored the role of needle diameter in microparticle delivery yield, and develop a modified viscosity formulation to improve microparticle delivery across a range of clinically relevant needle diameters. We have identified an optimal biocompatible formulation containing $0.25 \%$ pluronic F127 and $0.25 \%$ carboxymethyl cellulose, which can increase delivery payload to $520 \%$ across needle gauges 21-30G, and note that needle diameter impacts delivery efficacy. We use this formulation to increase the delivery yield of PLGA microparticles, and separately, PLGA-cell scaffolds supporting viable mesenchymal stem cells (MSCs), demonstrating the first in vitro delivery of this cell scaffold system. Together, these results highlight an optimal formulation for the delivery of microparticle and microparticle-cell scaffolds, and illustrate how careful choice of delivery formulation and needle size can dramatically impact delivery payload.
\end{abstract}

\section{Introduction}

Poly (DL-lactic acid-co-glycolic acid) (PLGA) materials are widely used therapeutics with applications in drug delivery (Rao et al., 2010; Rafati et al., 2012; Simon-Yarza et al., 2013), tissue engineering (Park et al., 2016), and cellular scaffolding (Lee et al., 2015; Qutachi et al., 2014). In drug delivery applications, PLGA microparticles offer tunable, biodegradable kinetic release profiles. They are FDA approved for a variety of applications, and can often be administered via localised or systemic injection (Rafati et al., 2012; Cappellano et al., 2014; Park et al., 2014). Larger 3-dimensional PLGA structures have also been surgically implanted as cellular scaffolds for regenerative medicine (Hernandez et al., 2010; Kochenderfer et al., 2010), however there are limited examples of systems combining the extracellular support matrix provided by PLGA (Lee et al., 2015; Han et al., 2015) with the tunable kinetic release of soluble factors (Cappellano et al., 2014). Through this combination of a physical support matrix and soluble cellular cues, microparticle cell scaffold systems with controlled release properties are able to support and direct transplanted cell behaviour.
A current challenge in the delivery of microparticles, cells, or microparticle-cell combination therapies lies in maintaining an effective therapeutic dose across varied application routes. In many systems, a common clinical administration route is the localised injection of materials using either pre-filled or self-filled syringes together with a needle (Garbayo et al., 2016; McHugh et al., 2015; Fu et al., 2016; Kim et al., 2016). Selection of an appropriate needle gauge depends on the therapeutic application; finer needles of $29 \mathrm{G}$ are often used for spatially accurate delivery of materials to the spinal cord, compared to $14 \mathrm{G}$ needles frequently used for intramuscular injection (Dittmann et al., 1993; Mavrogenis et al., 2015; Songur et al., 2011; Sivera et al., 2008; Raftesath and Fitzgerald, 2014). In cell-only systems, recent studies have suggested that injection parameters (including needle gauge, flow rate and applied force) can affect both the number of cells delivered and the ability of these cells to undergo phenotypic differentiation (Mamidi et al., 2012; Amer et al., 2016; Amer et al., 2015a,b), however these studies have yet to be applied to delivery of acellular microparticle systems.

Maintaining an effective therapeutic dose across a broad range of

\footnotetext{
* Corresponding author at: Division of Regenerative Medicine and Cellular Therapies, Centre for Biomolecular Sciences, School of Pharmacy, University of Nottingham, NG7 2RD, UK.

E-mail address: Derfogail.Delcassian@nottingham.ac.uk (D. Delcassian).
} 
administration routes remains an ongoing consideration for the clinical application of drug delivery systems (Cilurzo et al., 2011; Kearney and Mooney, 2013). To our knowledge, there has yet to be a study on the effect of injection parameters on delivery of either PLGA microparticles alone, or PLGA microparticles in conjunction with cells. Here, we explore modified viscosity systems to enhance both microparticle delivery and microparticle-cell scaffold delivery across a range of clinically relevant needle gauges. First, we explore PLGA particle delivery across a range of needle gauges with controlled plunger force. Next, we investigate modified delivery formulations using the thickening agent carboxymethylcellulose (CMC) (Nakanishi, 1966) and the amphiphilic polymer pluronic F127 (Prameela et al., 2015), probing their ability to modify viscosity and their effect on particle delivery across needle sizes. Finally, we investigate the effect of our lead formulation on the viability of human MSCs, and demonstrate the use of our formulation in delivering multifunctional PLGA microparticle scaffolds with human MSCs in vitro.

\section{Materials and methods}

\subsection{Fabrication of PLGA microparticles}

Non-porous PLGA particles were fabricated using 20\% PLGA (50:50, $52 \mathrm{kDa}$ Lakeshore Biomaterials) in dichloromethane (DCM) (Fischer) by either a single or double emulsion method. In the single emulsion method, the polymer solution was homogenised in $250 \mathrm{~mL}$ of $0.3 \%$ polyvinyl alcohol (13-24 kDa, Sigma-Aldrich) using a high speed Silverson L5M homogeniser. The resulting emulsion was left stirring at 300 RPM until particles hardened. In the double emulsion method, $100 \mu \mathrm{L}$ of an aqueous solution containing $10 \mathrm{mg}$ Amoxicillin (Abcam) was homogenised in the polymer solution. The resultant primary water in oil (w/o) emulsion was then homogenised again in the $0.3 \%$ PVA and the resultant water in oil in water $(\mathrm{w} / \mathrm{o} / \mathrm{w})$ double emulsion was left stirring until particles hardened. Particles were extracted by centrifugation, washed, and lyophilised before being stored at $-20^{\circ} \mathrm{C}$ until use. Porous PLGA particles were produced using a double emulsion method as previously described (Qutachi et al., 2014). Briefly, 20\% (w/ v) PLGA in dichloromethane was treated with phosphate buffered saline (PBS, Gibco) as a porogen. Post fabrication, the particles were treated with ethanolic sodium hydroxide (sodium hydroxide (Sigma-Aldrich) and absolute ethanol (Fischer)) to enhance surface porosity. The particles were then extracted by centrifugation, washed, and lyophilised before being stored at $-20^{\circ} \mathrm{C}$ until use.

Particles were characterised using scanning electron microscopy and laser diffraction. Briefly, particles were loaded onto carbon disks on aluminium stubs (Agar Scientific), sputter coated with gold (Balzers Union Ltd.) and imaged on an JEOL 6060L system. The mean diameter and particle size distribution were analysed using a Coulter LS230 particle size analyser (Beckman, UK). Particle size distribution was then determined as a function of the particle diffraction and plotted as a function of volume percentage.

\subsection{Delivery formulations}

Particles were resuspended at $5 \mathrm{mg} / \mathrm{mL}$ in DMEM (Gibco), containing between 0 and 10\% pluronic F127 (Sigma-Aldrich) or $0-10 \%$ medium viscosity sodium carboxymethylcellulose (CMC) (SigmaAldrich). Combined formulations containing between 0 and $0.5 \%$ pluronic and $0-0.5 \%$ CMC were also prepared. Formulation solutions were kept at $4{ }^{\circ} \mathrm{C}$ until use. Solution viscosity was measured using a rheometer with cone and plate geometry at $0.1^{\circ}$ angle (Anton ParrPhysica MCR 301)), using a shear ramp from 0 to $1001 / \mathrm{s}$ at $25^{\circ} \mathrm{C}$.

\subsection{Particle injection}

PLGA microparticles $(5.0 \mathrm{mg}$ ) were suspended in polymer/media formulation $(1.0 \mathrm{~mL})$ in $1.5 \mathrm{~mL}$ Eppendorf tubes under repeated pipetting and vortexing. The total volume was drawn up into a $1 \mathrm{~mL}$ disposable syringe (BD) and a needle (gauges 21G, 23G, 25G, 27G, 30G (BD Microlance)) fitted to the syringe prior to ejection of the total volume into a new Eppendorf tube. A sample $(10 \mu \mathrm{L})$ was taken from the ejected volume and particles were counted using a haemocytometer. For comparison, particles were also ejected through needles without a syringe to provide a control. Injections were considered to have failed when the contents of the syringe could not be ejected using a mechanically controlled syringe pump. This is usually due to a blockage in the needle or aggregation of the suspension at the syringe tip, resulting in the syringe contents not being completely emptied. Injection failures are recorded and measured in counts, and the calculated values illustrates the percentage of "failed" injections per condition.

\subsection{Injection forces and calculated shear rates}

For each needle-syringe combination, the initial and glide force were determined using a texture analyser (TA.HD plus, Stable micro systems). $1 \mathrm{~mL}$ of formulation was loaded into a $1 \mathrm{~mL}$ syringe (BD), and fitted with an appropriate needle into the injection rig. A $10 \mathrm{~mm}$ cylinder probe was lowered into contact with the plunger, with no pre-test force, before a $1 \mathrm{~mm} / \mathrm{s}$ ejection rate was applied in compression mode. The initial force was calculated as the force required to overcome the resistance to movement of the plunger, whereas the glide force was calculated as the average force required to evacuate the syringe at $1 \mathrm{~mm} / \mathrm{s}$. For formulations tested with microparticles, a concentration of $5 \mathrm{mg} / \mathrm{mL}$ particles suspended in $1 \mathrm{~mL}$ solution was used. Shear rates were calculated using Poiseuilles equation;

$\gamma=\frac{4 Q}{\pi r^{3}}$

where $\gamma$ is shear rate in $\mathrm{s}^{-1}, \mathrm{Q}$ is flow rate in $\mathrm{cm}^{3} / \mathrm{s}$, and $\mathrm{r}$ is needle radius in $\mathrm{cm}$. Shear rates were calculated using both experimental flow rate (for $1 \mathrm{~mm} / \mathrm{s}$ plunger ejection) and theoretical flow rates $1 \mathrm{~mL} / \mathrm{h}$ and $20 \mathrm{~mL} / \mathrm{h}$ expected to be used in clinic, described in Table 2.

\subsection{PLGA microparticle release studies}

In vitro testing of the controlled release of Amoxicillin encapsulated within PLGA microparticles was performed using Transwell inserts (Corning, UK). $25 \mathrm{mg}$ of PLGA microparticles were suspended in $1.5 \mathrm{~mL}$ of the described formulations, and incubated at $37^{\circ} \mathrm{C}$. The concentration of Amoxicillin in release medium was quantified by UV detection at $300 \mathrm{~nm}$ using a plate reader (Tecan) with concentration determined from a calibration curve.

\subsection{Cell viability}

Human bone marrow derived mesenchymal stem cells (MSCs) (UE6E7T-11 cells sourced from the Japanese Stem Cell Bank) were used for all cellular assays. The Prestoblue cell viability assay (Invitrogen Life Sciences, UK) was performed 1 and $24 \mathrm{~h}$ post-seeding $(n=6)$. Each sample was submerged in $1 \mathrm{~mL}$ of $10 \%$ Prestoblue (Invitrogen Life Sciences, UK) in media; all samples were incubated at $37^{\circ} \mathrm{C}$ for $30 \mathrm{~min}$. Triplicate $100 \mu \mathrm{L}$ media samples from each well were read on a Tecan plate reader with the excitation wavelength set to $535 \mathrm{~nm}$ and the emission wavelength set at $615 \mathrm{~nm}$.

\subsection{Injection of cells cultured on particles}

Porous particles were treated with Tween and then antibiotic/antimycotic solution (Sigma-Aldrich). Commercially available human mesenchymal stem cells (MSCs) (Japanese Stem Cell Bank) were seeded at 200,000 per well in 12-well plates, with $8 \mathrm{mg}$ PLGA particles added per well, and incubated overnight at $37^{\circ} \mathrm{C}$ in DMEM medium 
supplemented with $10 \%$ foetal calf serum, $1 \%$ antibiotic/antimycotic solution, $1 \%$ L-glutamine $(2 \mathrm{mM})$ and $1 \%$ non-essential amino acids (Sigma-Aldrich). Wells were centrifuged, and the cell pellet re-suspended in DMEM or formulation conditions. As described previously, this suspension was injected into a fresh 12-well plate, and incubated for 10 min with $10 \%$ Presto blue at $37^{\circ} \mathrm{C}$. Cell number per well was quantified using a Tecan plate reader. Samples were formalin fixed for 20 min at room temperature, washed with PBS multiple times and then imaged by SEM.

\subsection{Delivery efficacy}

Delivery efficacy was calculated by comparing the number of particles delivered using a specific formulation and needle combination to the number of particles delivered in a basal media solution. Particles were counted using a hemocytometer. For example, to compare the delivery efficacy of particles suspended in basal media through needlefree syringes and $27 \mathrm{G}$ needles, a suspension of PLGA particles was loaded into at least six identical syringes, three of which were uncapped and three capped with $27 \mathrm{G}$ needles. Syringes were loaded onto the controlled rate syringe pump, and ejected at constant plunger speed of $1 \mathrm{~mm} / \mathrm{s}$. An aliquot of the ejected solution was transferred to a hemocytometer and the number of ejected particles counted. To calculate delivery efficacy, we averaged the number of particles for each condition and calculated efficacy as follows;

delivery efficacy $=\frac{\text { average number of particles delivered in condition } X}{\text { average number of particles delivered in basal media }}$ $\times 100$

\subsection{Statistical analysis}

Statistical analysis was performed using GraphPad Prism (Version 7) software. ANOVA analysis was used for all statistical testing, performed on data from between 3 and 6 repeat experiments. Analysis is considered significant, and the * designation is assigned, if $\mathrm{p}>0.05^{*}$, $\mathrm{p}>0.01^{* *}, \mathrm{p}>0.001^{* * *}, \mathrm{p}>0.0001^{* * * *}$. Bar graphs represent the mean of 3-6 individual repeats, with associated error bars to show the standard error in the mean (SEM). Details of individual statistical tests (ie. one way or two way ANOVA, number of repeats) are provided in the figure caption for each graph.

\section{Results and discussion}

\subsection{The effect of needle gauge on microparticle delivery}

We fabricated PLGA microparticles of $27 \mu \mathrm{m}$ diameter as described in Section 2.1 (Fig. 1A), and investigated delivery efficacy through needles between 21 and 30G, corresponding to internal needle diameters currently used in clinic ranging from over $500 \mu \mathrm{m}$ to around $160 \mu \mathrm{m}$ (Table 1). Using a syringe pump set up with constant flow rate of $1 \mathrm{~mm} / \mathrm{s}$, we evaluated the ejection of PLGA microparticles suspended in basal media solution through a range of needle gauges (Fig. 1B). All needle gauges tested were able to deliver the microparticles, however we find that narrower needle gauges of $27 \mathrm{G}$ and $30 \mathrm{G}$ failed to deliver microparticle solutions as effectively as either needle free or large-bore needle systems, with delivery efficacy reduced to $61 \%$ in $27 \mathrm{G}$ needles compared to needle free systems. Given the internal needle diameters of $27 \mathrm{G}$ and $30 \mathrm{G}$ needles $(210 \mu \mathrm{m}$ and $160 \mu \mathrm{m}$ respectively) are wider than the $26.9 \mu \mathrm{m}$ ( \pm SD $11.2 \mu \mathrm{m}$ ) diameter of the microparticles (Fig. 1A), this suggests that the particle delivery through narrow gauge needles is affected not only by particle size, but also the dynamics of the fluid ejection from the syringe. To explore this further, we investigated the effect of altering the solution viscosity, and so ejection fluid dynamics, of the delivery formulation in needle delivery systems.

\subsection{PLGA microparticle delivery using modified formulations}

Formulations containing the thickening agent carboxymethylcellulose (CMC) (Fig. 1C), pluronic F127 (Fig. 1D) to modulate wettability, and CMC/pluronic F127 (Fig. 1E) combination formulations were tested for their ability to deliver PLGA microparticles. Fig. 1 demonstrates that increasing concentrations of either CMC or pluronic F127 in media significantly increased particle delivery, illustrated by the $400 \%$ increase of particle delivery in systems using either $5 \%$ pluronic F127 or 2\% CMC, compared to un-supplemented media. We then investigated combination formulations with compositions of between 0.125 and $0.5 \%$ of CMC and pluronic F127 in media. Fig. 1E illustrates that particle delivery was significantly improved in all combination formulations tested, and broadly increased as the concentration of CMC/pluronic F127 in solution increased.

We calculated the shear rate used during our experimental injections at a constant plunger rate of $1 \mathrm{~mm} / \mathrm{s}$ (Table 2). These values suggest our experimental injection system experiences low shear between 150 and 500/s. We examined the effect of formulation composition on viscosity at comparable shear rates between 1 and 100/s. Comparing the formulations, Fig. $1 \mathrm{~F}$ illustrates that the viscosity of each composition remains broadly constant against increasing shear between 1 and 100/s. In systems with constant CMC concentration and varying pluronic F127 concentration, viscosity measurements are similar, whilst viscosity measurements roughly double as the concentration of CMC doubles in the formulation, suggesting that viscosity properties are broadly driven by the CMC concentration in solution (Voigt et al., 2012; Ahmed et al., 2011). Given that many clinically administered injections are delivered at higher flow rates than those tested experimentally (Nguyen et al., 2002; Salman et al., 2017), we next calculated the expected flow rate and shear rate during more rapid administration through various needle gauges (Table 2). Needles injecting flow rates of $1 \mathrm{~mL} / \mathrm{h}$ and $20 \mathrm{~mL} / \mathrm{h}$ (flow rates more commonly used for clinical infusion regimes) experience much higher shear rates through similar needle gauges.

At the shear rates tested here, supplementing basal media with pluronic acid and CMC can increase microparticle delivery. There are several potential explanations for this, including the changes in solution viscosity and the increased wetting of microparticles in these formulations. The increased solution viscosity may help to form a stable microparticle suspension during syringe evacuation, which prevents particles being forced to the side of the syringe. Particles at the side of syringe can be statically attracted to the syringe casing and are less likely to be ejected (Whitaker et al., 2011). Additionally, Fig. 1E indicates that increasing the total polymer concentration to above $0.5 \%$, can increase delivery payload. It is likely that the negatively charged polymers help to combat static and attractive charges between the particles and the syringe, facilitating particle ejection (Park et al., 2014; Voigt et al., 2012). The combined effects of increased polymer concentration, particle wetting and increased viscosity, are likely responsible for the increase in delivery.

\subsection{Modified delivery formulations using clinical needle gauges}

All combination formulations tested demonstrated enhanced microparticle delivery compared to a basal media control in a $23 \mathrm{G}$ needle system. We then explored whether these combination formulations were able to enhance microparticle delivery across a range of clinically relevant needle gauges (Dittmann et al., 1993; Mavrogenis et al., 2015; Songur et al., 2011; Raftesath and Fitzgerald, 2014; Gill and Prausnitz, 2007; Fateh et al., 2014). Fig. 2A compares the number of microparticles delivered for each formulation in 21-27G needles, and Fig. 2B summarises the average delivery across all needle gauges tested. There is a significant increase in particle delivery across all needle gauges using CMC and pluronic F127 formulations in comparison to basal media ( $\mathrm{p}<0.001$ in all formulations, and all needle gauges, Turkey's 
A

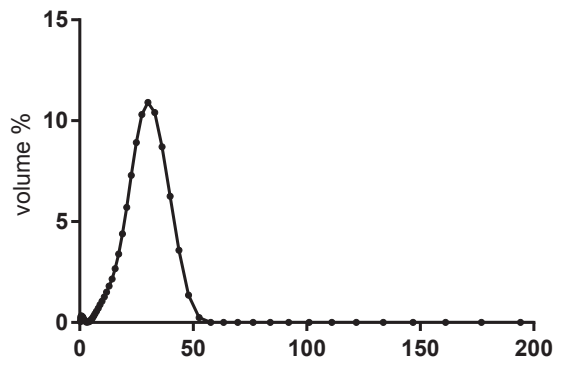

Particle diameter $(\mu \mathrm{m})$

$\mathrm{D}$

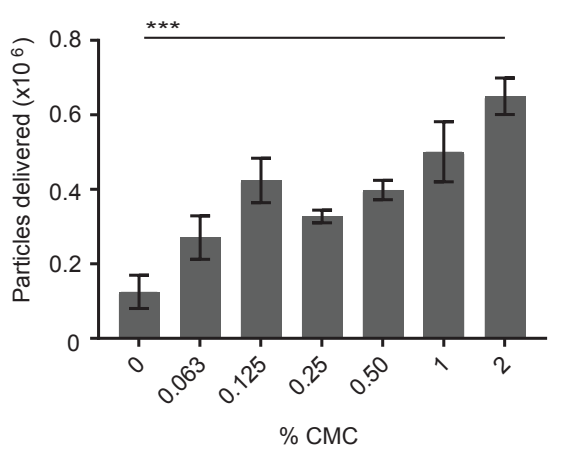

B

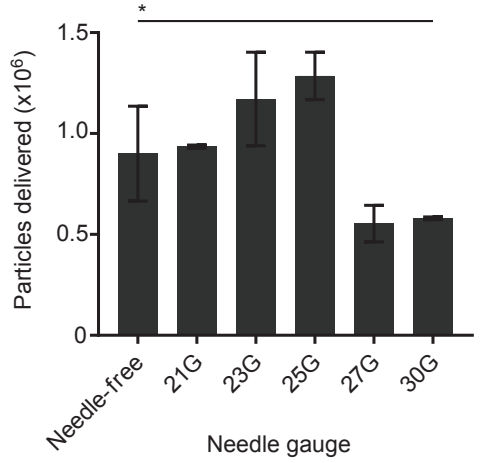

E

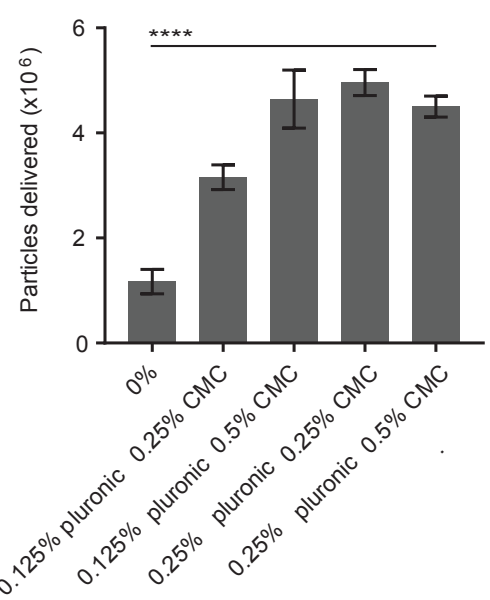

C

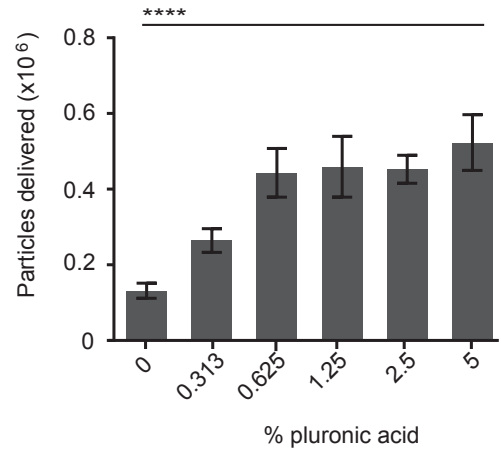

$\mathrm{F}$

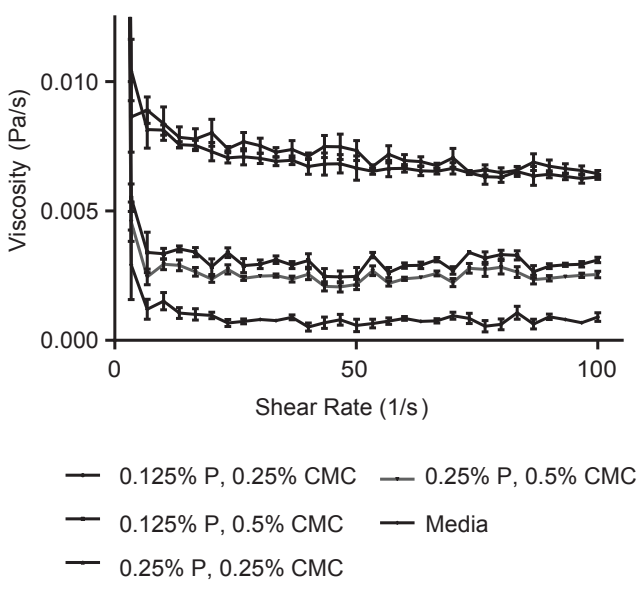

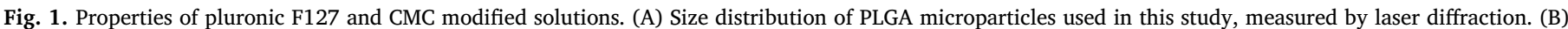

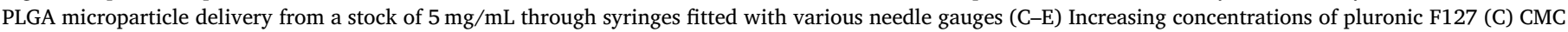

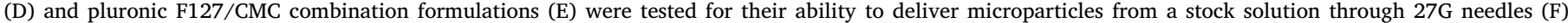

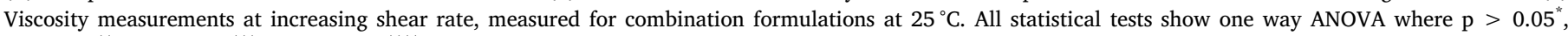
$\mathrm{p}>0.01^{* *}, \mathrm{p}>0.001^{* * *}, \mathrm{p}>0.0001^{* * * *}$, bars represent mean of 3-6 repeats with SEM error bars.

Table 1

Clinical needle gauges and needle bore internal diameters.

\begin{tabular}{llll}
\hline Needle Gauge & $\begin{array}{l}\text { Needle bore diameter } \\
(\mathrm{mm})\end{array}$ & Needle Gauge & $\begin{array}{l}\text { Needle bore diameter } \\
(\mathrm{mm})\end{array}$ \\
\hline 15 & 1.372 & 25 & 0.260 \\
16 & 1.194 & 26 & 0.260 \\
17 & 1.067 & 27 & 0.210 \\
18 & 0.838 & 28 & 0.184 \\
19 & 0.686 & 29 & 0.184 \\
20 & 0.603 & 30 & 0.159 \\
21 & 0.514 & 31 & 0.133 \\
22 & 0.413 & 32 & 0.108 \\
23 & 0.337 & 33 & 0.108 \\
24 & 0.311 & 34 & 0.0826 \\
\hline
\end{tabular}

multiple comparison tests and two-way ANOVA), with delivery increasing on average between 300 and $400 \%$ compared to particles delivered in basal media in the same needle gauge.

We further explored needle blockage and injection failure for each of these formulations (Fig. 2C) in needle gauges. Fig. 2C demonstrates that the $0.25 \%$ pluronic $\mathrm{F} 127$ and $0.25 \%$ CMC formulation had no injection failures compared to other formulations which occasionally resulted in a blocked needle injection failure. Considering this $0.25 \%$ pluronic F127 and $0.25 \%$ CMC formulation in more detail, we find that microparticle delivery is significantly increased in all needle gauges tested using this formulation compared to a basal media control (Fig. 2D), with increases in delivery between 320 and $750 \%$ compared
Table 2

Calculated shear rate through clinical needle gauges at varying flow. Shear rate was calculated using Poiseuilles equation for needle gauges between 21 and 30G, using experimental flow rates tested using $1 \mathrm{~mm} / \mathrm{s}$ plunger speed, and theoretical flow rates of 1 or $20 \mathrm{~mL} / \mathrm{h}$, all values rounded to 3 significant figures.

\begin{tabular}{|c|c|c|c|c|c|}
\hline \multirow[t]{2}{*}{$\begin{array}{l}\text { Needle } \\
\text { gauge }\end{array}$} & \multirow{2}{*}{$\begin{array}{l}\text { Needle } \\
\text { diameter } \\
\text { (cm) }\end{array}$} & \multicolumn{2}{|c|}{$\begin{array}{l}\text { Experimental flow } \\
\text { (plunger at } 1 \mathrm{~mm} / \mathrm{s} \text { ) }\end{array}$} & \multirow{2}{*}{$\begin{array}{l}\text { Theoretical } \\
\text { flow }(1 \mathrm{~mL} / \mathrm{h}) \\
\text { Shear rate } \\
\left(\mathrm{s}^{-1}\right)\end{array}$} & \multirow{2}{*}{$\begin{array}{l}\text { Theoretical } \\
\text { flow }(20 \mathrm{~mL} / \\
\text { h) } \\
\text { Shear rate } \\
\left(\mathrm{s}^{-1}\right)\end{array}$} \\
\hline & & $\mathrm{Q}\left(\mathrm{cm}^{3} / \mathrm{s}\right)$ & $\begin{array}{l}\text { Shear } \\
\text { rate } \\
\left(\mathrm{s}^{-1}\right)\end{array}$ & & \\
\hline $30 \mathrm{G}$ & 0.159 & $1.98 \mathrm{E}-07$ & 503 & 704,000 & $14,100,000$ \\
\hline $27 \mathrm{G}$ & 0.210 & $3.46 \mathrm{E}-07$ & 380 & 306,000 & $6,110,000$ \\
\hline $25 \mathrm{G}$ & 0.260 & $5.31 \mathrm{E}-07$ & 308 & 161,000 & $3,220,000$ \\
\hline $23 \mathrm{G}$ & 0.337 & $8.92 \mathrm{E}-07$ & 237 & 74,000 & $1,480,000$ \\
\hline $21 \mathrm{G}$ & 0.514 & $2.07 \mathrm{E}-06$ & 156 & 20,800 & 417,000 \\
\hline
\end{tabular}

to basal media (Table 3), and an average increase in microparticle delivery of $520 \%$ across all needle gauges. This formulation is therefore broadly applicable to a range of clinical needle gauges.

\subsection{Modified viscosity formulations for the delivery of drug loaded microparticles}

We investigated the applicability of the lead $0.25 \%$ CMC $0.25 \%$ pluronic F127 formulation for the delivery of drug-eluting microparticles and microparticle-cell scaffold therapeutics. First, the release 
A

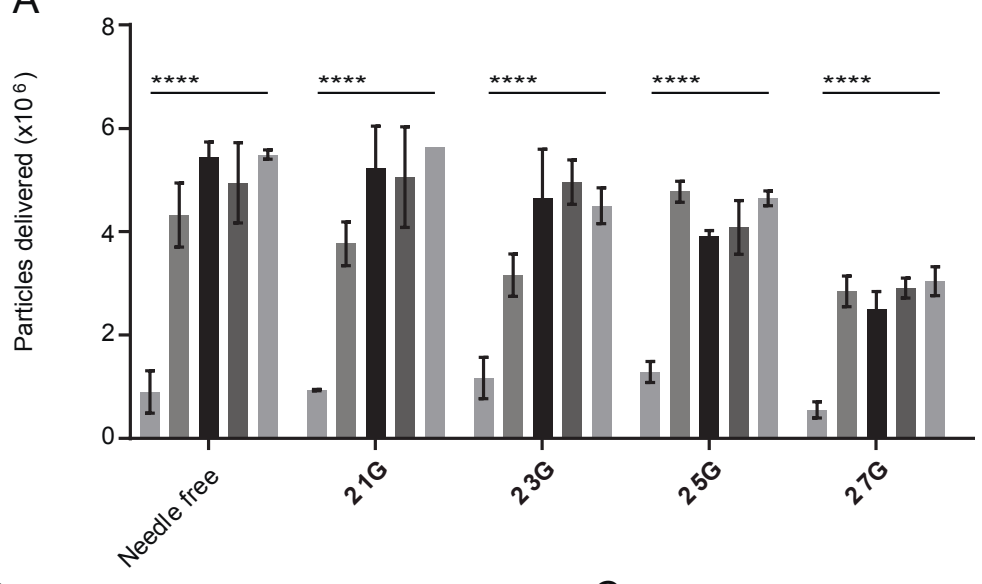

$\mathrm{B}$

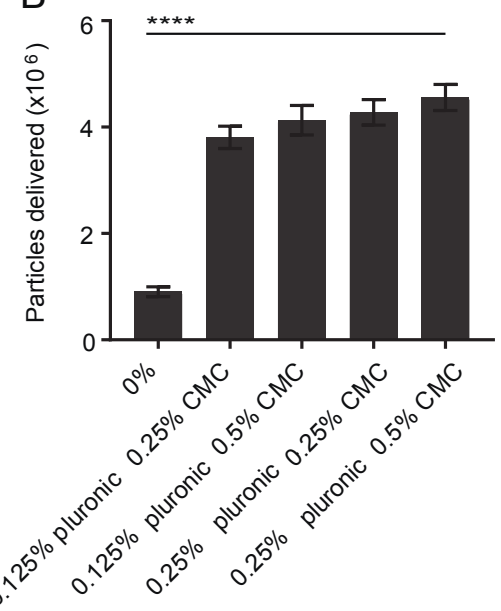

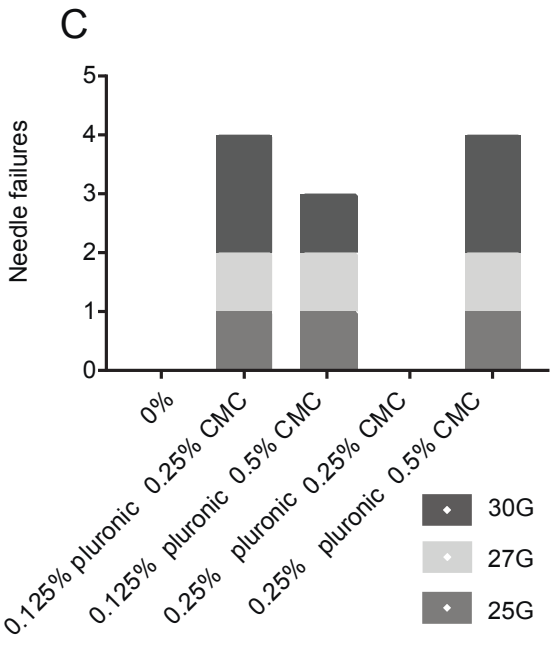

$0 \%$

$0.125 \%$ pluronic $0.25 \% \mathrm{CMC}$

$0.125 \%$ pluronic $0.5 \% \mathrm{CMC}$

$0.25 \%$ pluronic $0.25 \% \mathrm{CMC}$

$0.25 \%$ pluronic $0.5 \% \mathrm{CMC}$

Fig. 2. Modified solutions across needle gauges. Increasing concentrations of (A) pluronic F127/CMC combination formulations were tested for their ability to deliver microparticles from a stock solution across a range of needle gauges. (B) Average delivery of microparticles across all needle gauges (21-27G) in different combination formulations. (C) Number of needle blockages in each formulation, $\mathrm{n}=10$ for each formulation. Needles 30G, 27G, 25G, 23G and 21G were tested, with blockages found in 25-30G. (D) A comparison between basal media and $0.25 \%$ pluronic $0.25 \%$ CMC for the delivery of microparticles across needle gauges $21 \mathrm{G}-27 \mathrm{G}$. All statistical tests show two way ANOVA where $\mathrm{p}>0.05^{*}, \mathrm{p}>0.01^{* *}, \mathrm{p}>0.001^{* * * *}, \mathrm{p}>0.0001^{* * * * *}$, bars represent mean of 3-6 repeats with SEM error bars.

Table 3

Delivery efficacy Delivery efficacy was calculated by comparing the number of particles delivered. For example, PLGA microparticles were suspended in either basal media, or media supplemented with $0.25 \%$ CMC and $0.25 \%$ Pluronic F127, loaded into a standard syringe fitted with the appropriate gauge needle. Syringes were loaded onto the mechanically controlled syringe pump, and ejected. An aliquot of the ejected solution was transferred to a hemocytometer and the number of ejected particles counted. At least three syringe ejections were tested for each condition, and delivery efficacy calculated by comparing to the number of particles ejected in the basal media formulation.

\begin{tabular}{|c|c|c|c|c|}
\hline $\begin{array}{l}\text { Needle system and media } \\
\text { composition }\end{array}$ & $\begin{array}{l}\text { Average number of particles } \\
\text { delivered } / \mathrm{mL}\end{array}$ & $\begin{array}{l}\text { Compare to: Needle system and media } \\
\text { composition }\end{array}$ & $\begin{array}{l}\text { Average number of particles } \\
\text { delivered } / \mathrm{mL}\end{array}$ & $\begin{array}{l}\text { Calculated delivery efficacy } \\
(\%)\end{array}$ \\
\hline Needle free, basal media & $0.90 \times 10^{6}$ & $\begin{array}{l}\text { Needle free, } 0.25 \% \text { CMC } 0.25 \% \\
\text { pluronic F127 }\end{array}$ & $4.95 \times 10^{6}$ & $550 \%$ \\
\hline $21 \mathrm{G}$, basal media & $0.94 \times 10^{6}$ & $21 \mathrm{G}, 0.25 \%$ CMC $0.25 \%$ pluronic $\mathrm{F} 127$ & $5.06 \times 10^{6}$ & $538 \%$ \\
\hline 23G, basal media & $1.17 \times 10^{6}$ & $23 \mathrm{G}, 0.25 \%$ CMC $0.25 \%$ pluronic $\mathrm{F} 127$ & $4.96 \times 10^{6}$ & $424 \%$ \\
\hline 25G, basal media & $1.29 \times 10^{6}$ & $25 \mathrm{G}, 0.25 \%$ CMC $0.25 \%$ pluronic $\mathrm{F} 127$ & $4.08 \times 10^{6}$ & $316 \%$ \\
\hline $27 \mathrm{G}$, basal media & $0.55 \times 10^{6}$ & $27 \mathrm{G}, 0.25 \%$ CMC $0.25 \%$ pluronic $\mathrm{F} 127$ & $2.91 \times 10^{6}$ & $529 \%$ \\
\hline 30G, basal media & $0.58 \times 10^{6}$ & $30 \mathrm{G}, 0.25 \%$ CMC $0.25 \%$ pluronic F127 & $4.37 \times 10^{6}$ & $753 \%$ \\
\hline
\end{tabular}

profile of amoxicillin from amoxicillin loaded PLGA microparticles was tested, exploring microparticles delivered through a $27 \mathrm{G}$ needle with and without the modified viscosity formulation (Fig. 3A). As expected, the total amount of amoxicillin released was increased when our delivery formulation was used. As the kinetics of release remained broadly unaffected, we suggest that this is due to the increased delivery yield using the $0.25 \%$ CMC $0.25 \%$ pluronic F127 formulation.

Interestingly, the addition of pluronic acid and CMC may also alter microparticle surface wetting and shear forces experienced during ejection, which could also influence drug release kinetics. The hydrophilicity of PLGA surfaces affects polymer degradation kinetics, and is correlated with both co-polymer composition and liquid solvent polarity (Chen et al., 2017; Vargha-Butler et al., 2001). The addition of CMC and pluronic acid to the basal media formulation may alter the polarity of the liquid phase, and could directly impact microparticle surface wetting and so drug release kinetics. Formulations containing CMC and pluronic acid have also been shown to alter shear forces experienced by cells during stirred culture (Gallardo Rodriguez et al., 
A

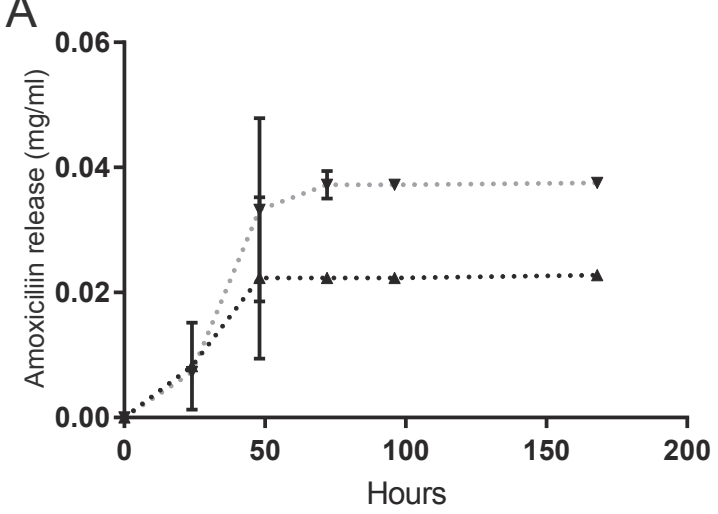

-. $27 \mathrm{G}$ needle

v. $27 \mathrm{G}$ needle $(0.25 \%$ Pluronic, $0.25 \% \mathrm{CMC})$
B

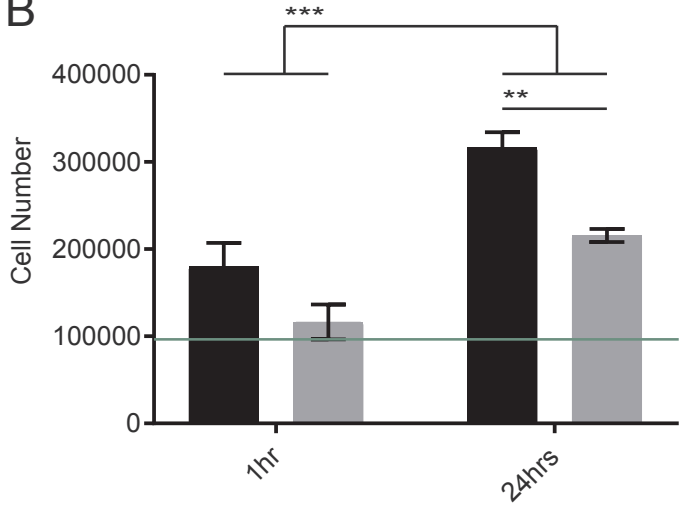

$0 \% \square 0.25 \%$ pluronic $0.25 \% \mathrm{CMC}$ — Seeding density
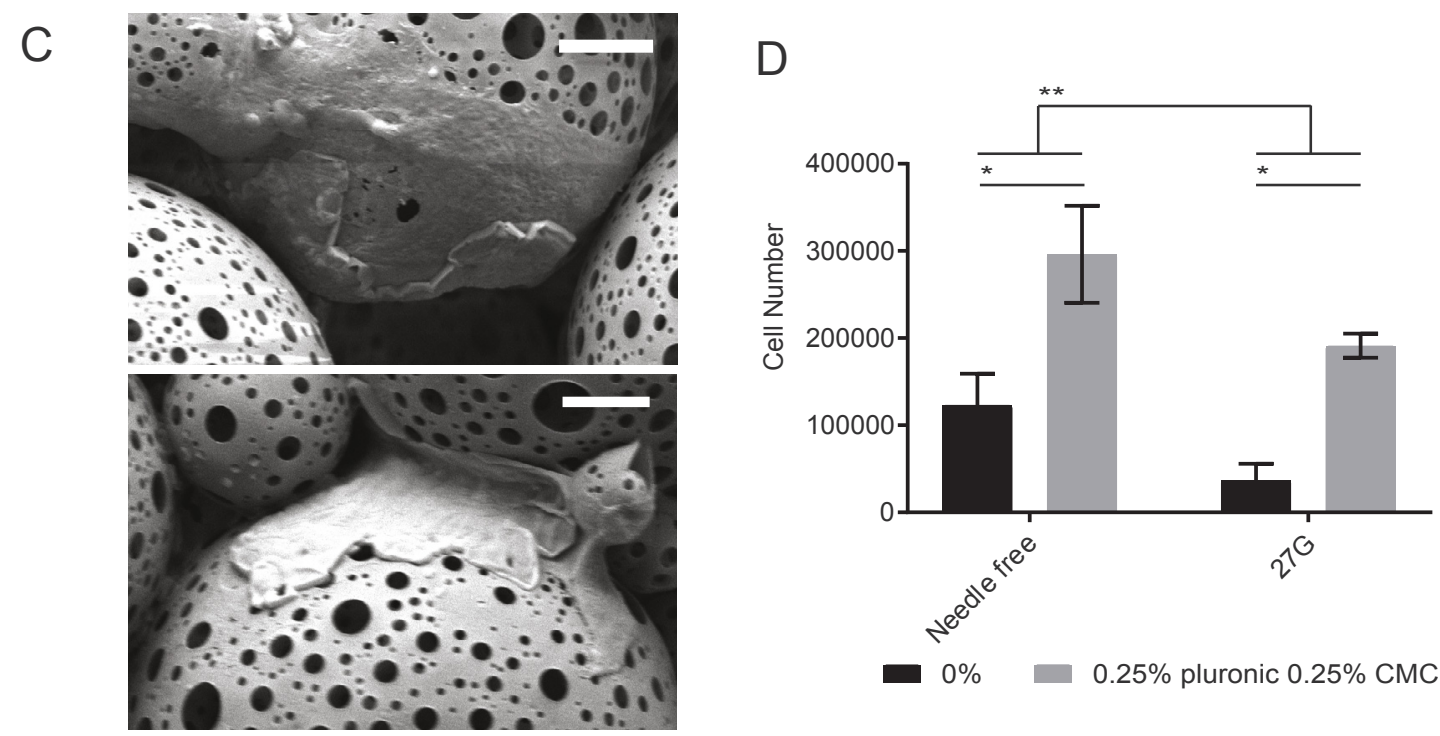

Fig. 3. Effect of modified formulations on drug release and cell behaviour (A) Cumulative amoxicillin release was analysed from PLGA microparticles delivery using syringes fitted $27 \mathrm{G}$ needles. PLGA microparticles were suspended in basal media or $0.25 \%$ pluronic $0.25 \%$ CMC supplemented media (C) SEM image of microparticle cell scaffold post-delivery through a $27 \mathrm{G}$ needle. Scale bar $10 \mu \mathrm{m}$. (B, D) Cell number after cellular incubation in basal media, or media supplemented with $0.25 \%$ CMC and $0.25 \%$ pluronic F127 after 1 and $24 \mathrm{~h}$ compared to an initial seeding density (B), (D) Cell number post simulated delivery through a needle free of $27 \mathrm{G}$ needle system using basal media or media supplemented with $0.25 \% \mathrm{CMC}$ and $0.25 \%$ pluronic F127. Statistical analysis performed using ANOVA, all statistical tests show $\mathrm{p}>0.05^{*}, \mathrm{p}>0.01^{* * *}, \mathrm{p}>0.001^{* * * *}, \mathrm{p}>0.0001^{* * * * *}$, bars represent mean of 3-6 repeats with SEM error bars.

2011; Xu et al., 1995). The addition of these molecules to the formulation may therefore impact the shear forces particles are exposed to during ejection. To explore the relative importance of particle delivery yield, surface wetting, and ejection shear in controlling drug delivery, further studies should be performed to independently isolate these variables.

\subsection{Modified viscosity formulations for the delivery of microparticles-cell scaffolds}

Next, we investigated the effect of our modified viscosity formulation on cell viability. At low concentrations, both CMC and pluronic acid moieties have been shown to protect cells from detrimental effects due to excessive shear forces (Gallardo Rodriguez et al., 2011; Xu et al., 1995). We examined the viability of human mesenchymal stem cells (MSCs) at $1 \mathrm{~h}$ and $24 \mathrm{~h}$ in media supplemented with our formulation. Fig. 3B shows cell proliferation calibrated using the Presto Blue metabolic assay in both conditions. In both solutions, cells demonstrate a similar viability at $1 \mathrm{~h}$, and at $24 \mathrm{~h}$ show a significant increase in metabolic activity compared to $1 \mathrm{~h}$. Cells remained viable in the formulation for up to $24 \mathrm{~h}$, though there was a reduction in metabolic activity after incubation for $24 \mathrm{~h}$ in our formulation compared to basal media. At the $1 \mathrm{~h}$ timepoint, which represents a realistic timeframe for the clinical administration of cell-particle systems, there was no significant difference in viability between cells cultured in basal media and the $0.25 \%$ pluronic F127 $0.25 \%$ CMC formulation, indicating this formulation may be suitable for the in vivo delivery of microparticle-cell systems.

Finally, we investigated the delivery of cells together with PLGA microparticles through syringes, either with or without a $27 \mathrm{G}$ needle, in basal media or our $0.25 \%$ CMC and $0.25 \%$ pluronic F127 formulation. Fig. 3C shows SEM images of cells delivered together with PLGA microparticle scaffolds, and demonstrates the integrity of both the particles and the cells post injection, and that the PLGA particles can be used to provide a scaffold for the cells. Fig. 3D illustrates that in both needle free and $27 \mathrm{G}$ needle delivery systems, the use of the $0.25 \% \mathrm{CMC}$ and $0.25 \%$ pluronic F127 formulation significantly increased cell delivery by $200-400 \%$ compared to basal media. These results are comparable 
to our earlier studies demonstrating enhanced delivery in microparticle only systems, and support our conclusion that basal media supplemented with $0.25 \% \mathrm{CMC}$ and $0.25 \%$ pluronic $\mathrm{F} 127$ provides a formulation that can enhance delivery of PLGA microparticles, and microparticle-cell combination therapeutics, in needle delivery systems without compromising cell viability. We believe these results show the first needle delivery of a porous PLGA microparticle-MSC system, as proof-of-concept drug-eluting microparticle-cell scaffolds capable of combining a biodegradable cell support with localised drug delivery. Encapsulating soluble factors which direct host- or transplanted cell behaviour within these microparticle scaffolds would increase their versatility and make them a powerful tool for cell transplant.

\section{Conclusion}

The delivery of a controlled dose of microparticles is crucial for therapeutic applications. We find that the addition of viscosity modifiers can enhance particle delivery up to $520 \%$ across needle gauges between 21 and 30G, and identify a formulation of basal media supplemented with $0.25 \%$ pluronic $\mathrm{F} 127$ and $0.25 \%$ CMC as providing an optimal system. Although the polymers explored here increase delivery across all needle sizes tested, our results indicate that selection of an appropriate needle is also an important parameter to consider. We tested the biocompatibility of our lead formulation, finding that cells remain viable in the formulation for up to $24 \mathrm{~h}$, and demonstrate that this formulation is suitable for the improved in vitro delivery of drug eluting PLGA microparticles, and microparticle-cell scaffolds. These microparticle-cell scaffolds offer the potential to simultaneously support cells for transplant and modulate the host environment/transplanted cell behaviour through the controlled release of pharmaceuticals. Together, these results pave the way for further exploration of microparticle-cell scaffold and delivery systems for in vivo cell transplantation. Additionally, these results have important implications for the application of microparticle and microparticle-cell therapeutics, and may also apply to other polymer based pharmaceuticals or protein biologics delivered by needle. In many cases, in vitro testing and clinical applications use different delivery strategies, with different needle and formulation systems, which could lead to differences in administered therapeutic dose. In order to match in vitro, pre-clinical and therapeutic outcomes, administration parameters (such as needle diameter and delivery formulation) should be carefully considered, and ideally conserved between pre-clinical and therapeutic applications.

\section{Acknowledgements}

We would like to acknowledge funding provided by the UKRMP Acellular Hub and the EPSRC E-TERM program, which helped to support this work.

\section{References}

Ahmed, T.A., et al., 2011. In vitro release, rheological, and stability studies of mefenamic acid coprecipitates in topical formulations. Pharm. Dev. Technol. 16 (5), 497-510.

Amer, M.H., et al., 2015b. Evaluation of delivery of mesenchymal stem cells using smallgauge needles: tailoring administration of cell-based therapies for efficient clinical translation. Tissue Eng. Part A 21, S265.

Amer, M.H., et al., 2016. A detailed assessment of varying ejection rate on delivery ef ficiency of mesenchymal stem cells using narrow-bore needles. Stem Cells Transl. Med. 5 (3), 366-378.

Amer, M.H., White, L.J., Shakesheff, K.M., 2015a. The effect of injection using narrowbore needles on mammalian cells: administration and formulation considerations for cell therapies. J. Pharm. Pharmacol. 67 (5), 640-650.

Cappellano, G., et al., 2014. Subcutaneous inverse vaccination with PLGA particles loaded with a MOG peptide and IL-10 decreases the severity of experimental autoimmune encephalomyelitis. Vaccine 32 (43), 5681-5689.

Chen, W.L., et al., 2017. Effect of particle size on drug loading and release kinetics of gefitinib-loaded PLGA microspheres. Mol. Pharm. 14 (2), 459-467.

Cilurzo, F., et al., 2011. Injectability evaluation: an open issue. AAPS PharmSciTech 12
(2), 604-609.

Dittmann, M., et al., 1993. 9 Years of clinical-experience with 29 gauge spinal needles. Br. J. Anaesthesia 70, 65.

Fateh, M., et al., 2014. Syringe-type and needle gauge have no role in adverse events following DTwP immunization: a randomized multicenter trial. Pediatric Infect. Dis. J. 33 (9), E239-E246.

Fu, J., et al., 2016. Subconjunctival delivery of dorzolamide-loaded poly(ether-anhy dride) microparticles produces sustained lowering of intraocular pressure in rabbits. Mol. Pharm. 13 (9), 2987-2995.

Gallardo Rodriguez, J.J., et al., 2011. Carboxymethyl cellulose and Pluronic F68 protect the dinoflagellate Protoceratium reticulatum against shear-associated damage. Bioprocess Biosyst. Eng. 34 (1), 3-12.

Garbayo, E., et al., 2016. Catheter-based intramyocardial injection of FGF1 or NRG1loaded MPs improves cardiac function in a preclinical model of ischemia-reperfusion. Sci. Rep. 6.

Gill, H.S., Prausnitz, M.R., 2007. Does needle size matter? J. Diabetes Sci. Technol. 1 (5), 725-729.

Han, K.-S., et al., 2015. Effect of demineralized bone particle/poly(lactic-co-glycolic acid) scaffolds on the attachment and proliferation of mesenchymal stem cells. J. Biomater. Sci. Polym. Ed. 26 (2), 92-110.

Hernandez, R.M., et al., 2010. Microcapsules and microcarriers for in situ cell delivery. Adv. Drug Deliv. Rev. 62 (7-8), 711-730.

Kearney, C.J., Mooney, D.J., 2013. Macroscale delivery systems for molecular and cellular payloads. Nat. Mater. 12 (11), 1004-1017.

Kim, Y.-C., et al., 2016. Transplantation of mesenchymal stem cells for acute spinal cord injury in rats: comparative study between intralesional injection and scaffold based transplantation. J. Korean Med. Sci. 31 (9), 1373-1382.

Kochenderfer, J.N., et al., 2010. A phase I clinical trial of treatment of B cell malignancies with autologous anti CD19 CAR transduced T cells. Blood 116 (21), 1179-1180.

Lee, Y.S., et al., 2015. Development of porous PLGA/PEI1.8k biodegradable microspheres for the delivery of mesenchymal stem cells (MSCs). J. Controll. Release 205, $128-133$.

Mamidi, M.K., et al., 2012. Impact of passing mesenchymal stem cells through smaller bore size needles for subsequent use in patients for clinical or cosmetic indications. J. Transl. Med. 10, 229.

Mavrogenis, G., et al., 2015. 25-gauge histology needle versus 22-gauge cytology needle in endoscopic ultrasonography-guided sampling of pancreatic lesions and lymphadenopathy. Endosc. Int. Open 3 (1), E63-E68.

McHugh, K.J., et al., 2015. Single-injection vaccines: progress, challenges, and opportunities. J. Controll. Release 219, 596-609.

Nakanishi, Y., 1966. Studies on pharmaceutical suspensions. 3. Viscosities of the dispersed systems of barium sulfate in MC and CMC aqueous solutions. Yakugaku Zasshi 86 (11), 997-1000.

Nguyen, N.T., Huang, X.Y., Chuan, T.K., 2002. MEMS-micropumps: a review. J. Fluids Eng. Trans. ASME 124 (2), 384-392.

Park, C.H., et al., 2014. Needle-free transdermal delivery using PLGA nanoparticles: Effect of particle size, injection pressure and syringe orifice diameter. Colloids Surf. B: Biointerfaces 123, 710-715.

Park, J.S., et al., 2016. Stem cell differentiation-related protein-loaded PLGA microspheres as a novel platform micro-typed scaffold for chondrogenesis. Biomed. Mater. 11 (5).

Prameela, G.K., et al., 2015. Physicochemical perspectives (aggregation, structure and dynamics) of interaction between pluronic (L31) and surfactant (SDS). Phys. Chem. Chem. Phys. 17 (45), 30560-30569.

Qutachi, O., et al., 2014. Injectable and porous PLGA microspheres that form highly porous scaffolds at body temperature. Acta Biomater. 10 (12), 5090-5098.

Rafati, A., et al., 2012. Chemical and spatial analysis of protein loaded PLGA microspheres for drug delivery applications. J. Controll. Release 162 (2), 321-329.

Raftesath, D., Fitzgerald, M., 2014. Influence of needle gauge used for venipuncture on automated platelet count and coagulation profile in dogs. Aust. Vet. J. 92 (3), N16.

Rao, D.A., et al., 2010. Biodegradable PLGA based nanoparticles for sustained regional lymphatic drug delivery. J. Pharm. Sci. 99 (4), 2018-2031.

Salman, D., et al., 2017. Evaluation of the performance of elastomeric pumps in practice: are we under-delivering on chemotherapy treatments? Curr. Med. Res. Opin. 33 (12), 2153-2159.

Simon-Yarza, T., et al., 2013. PEGylated-PLGA microparticles containing VEGF for long term drug delivery. Int. J. Pharm. 440 (1), 13-18.

Sivera, F., Aragon, R., Pascual, E., 2008. First metatarsophalangeal joint aspiration using a 29-gauge needle. Ann. Rheumatic Dis. 67 (2), 273-275.

Songur, N., et al., 2011. Comparison of 19-and 22-gauge needles in EUS-guided fine needle aspiration in patients with mediastinal masses and lymph nodes. Turk. J. Gastroenterol. 22 (5), 472-478.

Vargha-Butler, E.I., et al., 2001. Wettability of biodegradable surfaces. Colloid Polym. Sci. 279 (12), 1160-1168.

Voigt, M., Koerber, M., Bodmeier, R., 2012. Improved physical stability and injectability of non-aqueous in situ PLGA microparticle forming emulsions. Int. J. Pharm. 434 (1-2), 251-256.

Whitaker, M.A., et al., 2011. Particle size and shape effects in medical syringe needles: experiments and simulations for polymer microparticle injection. J. Mater. Sci. Mater. Med. 22 (8), 1975-1983.

Xu, D., et al., 1995. Studies of protective properties of pluronic and other agents on the hybridoma cell culture. Chin. J. Biotechnol. 11 (2), 101-107. 